\title{
Strongly Red-Shifted Photoluminescence Band Induced by Molecular Twisting in Cyanine (Cy3) Dye Films
}

Surendra B. Anantharaman, ${ }^{\dagger, \S}$ Sergii Yakunin, ${ }^{\dagger, \perp}{ }^{\infty}$ Chuyao Peng, ${ }^{\dagger}$ Marcus Vinícius Gonçalves Vismara, ${ }^{\#(\mathbb{C}}$ Carlos F. O. Graeff, ${ }^{\#}$ Frank A. Nüesch, ${ }^{\dagger, \S}$ Sandra Jenatsch, ${ }^{\dagger, \S}$ Roland Hany, ${ }^{\dagger \odot}$ Maksym V. Kovalenko, ${ }^{\dagger, \perp}{ }_{\odot}$ and Jakob Heier* ${ }^{*}+$ (i)

${ }^{\dagger}$ Laboratory for Functional Polymers and ${ }^{\ddagger}$ Laboratory for Thin Films and Photovoltaics, Swiss Federal Laboratories for Materials Science and Technology (Empa), Überlandstrasse 129, CH-8600 Dübendorf, Switzerland

${ }^{\S}$ Institut des matériaux, École Polytechnique Fédérale de Lausanne EPFL, Lausanne CH-1015, Switzerland

${ }^{\perp}$ Laboratory of Inorganic Chemistry, Department of Chemistry and Applied Biosciences, ETH Zurich, CH-8093 Zürich, Switzerland

${ }^{\#}$ Faculdade de Ciências, Universidade Estadual Paulista Júlio de Mesquita Filho (UNESP), 17033-360 Bauru, Brazil

\section{Supporting Information}

ABSTRACT: Cyanine dye molecules, used as monomers or in aggregate form, find interesting applications in optoelectronic devices. Among the various aggregate species incorporating organic dyes, centrosymmetric dimers are known as nonluminescent. They can act as exciton quenchers due to a low-energy optically forbidden excited state. In this study, however, we show that a dimer species in thin films exhibits efficient and strongly red-shifted photoluminescence. When the films were excited, a monomer emission at $590 \mathrm{~nm}$ along with a second emission peak at $680 \mathrm{~nm}$ was observed. A close relation between the dye concentration and the emission showed that a new emission at $680 \mathrm{~nm}$ corresponds to the dimer emission. Circular dichroism (CD) spectroscopy reveals that a fraction of the dimers exist in a twisted dimer configuration. Stable, long-lived, and quenchable fluorescence with high quantum yield is attributed to this dimer emission.

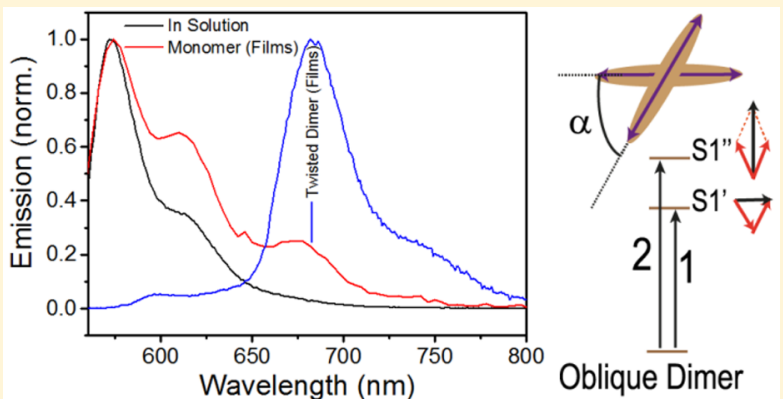

\section{INTRODUCTION}

Organic dyes such as oxazines, rhodamines, cyanines, and merocyanines are used as energy transport medium in various applications including optoelectronic devices, light harvesting antennas, photography, and dye lasers. ${ }^{1,2}$ Monomer emission is strongly diminished when surrounded by aggregate trap states (aggregation-caused quenching, ACQ). ${ }^{3}$ Absorption and fluorescence of these dye molecules in different molecular configurations (dimers, H- or J-type) are dictated by the net transition dipole moments of the optical transitions between ground state $S_{0}$ and excited state $S_{n}$ and largely follow Kasha's rule. ${ }^{4}$ In homodimers and $\mathrm{H}$-aggregates, the net transition dipole moment is zero, which renders them nonfluorescent. However, transitions to/from the excited $\left(S_{1}{ }^{\prime}\right)$ state are fully forbidden only for a perfect parallel or antiparallel alignment of dye molecules. 5

It is highly desirable in this context to learn more about $\mathrm{H}$ aggregate dimer trap states and their role in excited-state deactivation in organic amorphous films. Highly luminescent $\mathrm{H}$-aggregates and dimers have been reported in solution ${ }^{6-8}$ and thin films. ${ }^{9,10}$ This phenomenon was ascribed to the small distortion between two dye molecules along the chromophore axis resulting in the rotational twist $(\alpha)$ by $10^{\circ}$. Rigidification of these twisted dye molecules in the solid state can result in an enhancement of fluorescence, ${ }^{11}$ obviating the effect of ACQ and forbidden transitions.

Cyanines, rhodamine, and rubrene have shown anomalous photoluminescence (PL) bands in the solid state. ${ }^{12-14}$ For example, PL was studied for pseudoisocyanine dyes adsorbed into microcrystalline cellulose. ${ }^{12}$ Upon excitation at $532 \mathrm{~nm}$, a new emission peak at $680 \mathrm{~nm}$ was observed in these films along with a monomer emission $(620 \mathrm{~nm})$. The emission intensity scaled well with the dye concentration and excitation intensity. The authors hypothesized that the emission at $680 \mathrm{~nm}$ could be due to dimer formation at high laser fluences. In contrast to this, there are also reports indicating triplet formation in cyanine dimers, which can lead to this second emission. ${ }^{15-17}$ Similarly, the formation of excimers has been observed. ${ }^{18}$ It is still unclear what the underlying mechanisms are for additional emission bands in the films.

In the present study, a detailed investigation is carried out using different spectroscopic techniques such as electron spin resonance (ESR), CD, and fluorescence to identify the origin of an unexpected emission in the Cy3 dye $1,1^{\prime}$-diethyl-3,3,3', $3^{\prime}$ -

Received: February 13, 2017

Revised: April 11, 2017

Published: April 17, 2017 
tetramethylcarbocyanine. In the thin film, an emission band at $680 \mathrm{~nm}$ (red-shifted by $100 \mathrm{~nm}$ from the monomer band) is significantly more intense than the monomer emission. However, this emission is not observed in solution. Our investigations support the hypothesis that the second emission originates from dimers in the sample that are present in a twisted dimer configuration. The fraction of dimers in that configuration is low. Guidelines for achieving high quantum efficiency of dimers compared to the normal monomer emission and the possibility to harvest this emission is presented. The development of efficient solid state emitters is a prospering research field as a result of the numerous technological applications employing luminescent materials in thin films. ${ }^{19}$

\section{EXPERIMENTAL PROCEDURE}

Materials. Trimethine cyanine dyes (Cy3) 1-ethyl-2-[3-(1ethyl-1,3-dihydro-3,3-dimethyl-2H-indol-2-ylidene)-1-propen1-yl]-3,3-dimethyl-3H-indolium with the counterions hexafluorophosphate (Cy3-P), iodide (Cy3-I), and tetraphenylborate (Cy3-B) were procured from FEW Chemicals, Germany. Two counterions, perchlorate (Cy3-C) and $\Delta$-TRISPHAT (Cy3-T), accompanying the same chromophore were synthesized in our laboratory as reported elsewhere. ${ }^{20}$ Trimethine dyes 3-ethyl-2[3-(3-ethyl-3H-benzothiazol-2-ylidene)propenyl] benzoazol-3ium perchlorate and 3-ethyl-2-[3-(3-ethyl-3H-benzooxazol-2ylidene)propenyl]benzothiazol-3-ium perchlorate were purchased from FEW Chemicals, Germany.

The purity of the synthesized dye were verified by NMR spectroscopy (not shown here). The highest purity dyes procured from FEW chemicals showed similar results. Suitable solvents were chosen to dissolve the dyes along with two different polymers, either poly(methyl vinyl ether) (PVME) or poly(methyl methacrylate) (PMMA), separately.

Film Formation. Glass substrates were cleaned in acetone, ethanol, and Hellmanex solutions before spin-coating with the dye solution. The dye solution was spin-coated at $2000 \mathrm{rpm}$ for 2 min on glass substrate to obtain a homogeneous film. For fluorescence quenching studies, the dye was blended with $\mathrm{NOPF}_{6}$ in the solution and spin-coated on the glass substrates.

Characterizations. The absorption spectra for the films were acquired using a Varian Cary $50 \mathrm{UV}$-vis spectrometer. Fluorescence spectroscopy (Horiba Jobin Yvon Fluorolog equipped with integrating sphere) was used to obtain the excitation and emission scans from the dye films. Fluorescence quantum efficiencies were measured following the procedure of deMello. ${ }^{21}$ Fluorescence quenching was studied for the films deposited from the blend of dye $/ \mathrm{NOPF}_{6}$ to determine the characteristics of energy transfer. Subsequently, temperaturedependent fluorescence studies were conducted for the dye films to determine the role of a thermally activated process in the emission. Spin-coated films were mounted into a self-built cooling station facilitated with an adiabatic compression cooling to reach the desired temperature. Fluorescence signals were collected at different intervals while monitoring the substrate temperature when cooling and heating the sample between room temperature and $92 \mathrm{~K}$. The lifetime of the emission signal was monitored at room temperature using the Quantaurus-Tau fluorescence lifetime spectrometer from Hamamatsu Photonics, K.K. Japan. A xenon lamp source with lamp intensity of $4 \mathrm{~mW}$ was used to excite the film at different wavelengths, and fluorescence signals were collected with a time resolution of 0.1 ns. The lifetime and emission wavelength can be used to classify the role of different species present in the film. To further understand the stability of the signals to light and oxygen, the processed films were kept in a nitrogen environment and stored in the dark and in daylight separately for 30 days. At different time intervals, the emission from these samples was monitored to determine the stability of the emitting species. Circular dichroism spectroscopy (JASCO J-815 CD spectrometer) was used to characterize the spin-coated films at room temperature from 400 to $650 \mathrm{~nm}$ with a scan rate of $100 \mathrm{~nm} / \mathrm{min}$ and pitch for $0.2 \mathrm{~nm}$.

Cyanine dyes Cy3-I, Cy3-C, Cy3-P, and Cy3-T were studied by electron spin resonance (ESR) and by light-induced electron spin resonance (LESR) at temperatures from 77 to $298 \mathrm{~K}$. In addition, Cy3-T was studied at a temperature of $6.5 \mathrm{~K}$. For the LESR measurements with temperature between 77 and $298 \mathrm{~K}$, a Miniscope MS300 spectrometer from Magnettech (X-band and 9.3-9.55 GHz) was used. For measurements at $6.5 \mathrm{~K}$, a Bruker Elexsys E580 spectrometer (X-band) was used, and the temperature was controlled by a continuous flow of liquid helium using an Oxford cryogenic system, model ITC503. The microwave power used was between 0.100 and $31.62 \mathrm{~mW}$. Samples were illuminated by a $140 \mathrm{~W}$ xenon lamp from a Newport solar simulator using an AM 1.5 filter. The samples were illuminated inside the ESR cavity. Diphenylpicrylhydrazyl (DPPH) with $g=2.0036$ was used as $g$-factor reference. The ESR spectra were simulated using the software EasySpin implemented in MATLAB (MathWorks, Inc.).

\section{RESULTS AND DISCUSSION}

Cy3 dyes with different counterions like $\Delta$-TRISPHAT (Cy3$\mathrm{T})$, iodide (Cy3-I), hexafluorophosphate (Cy3-P), and tetraphenyl borate (Cy3-B) were coated from suitable solvents to prepare thin films. Cy3-T in chlorobenzene $(\mathrm{CB})$ gives a monomer absorption peak at $559 \mathrm{~nm}$ and a vibronic peak at $526 \mathrm{~nm}$ (Figure 1a). Upon excitation at $550 \mathrm{~nm}$, an emission was observed at $572 \mathrm{~nm}$, which is assigned to monomer fluorescence in solution. Spin-coated films prepared from the same dye solution show monomer and dimer absorptions at 577 and $529 \mathrm{~nm}$, respectively. In the PL spectra, the peak at $588 \mathrm{~nm}$ is assigned to monomer emission. In solution and in films, a vibrational shoulder to the monomer peak was observed at higher wavelength. However, the dye films show a strong dimer emission at $680 \mathrm{~nm}$, which is red-shifted from the monomer peak.

The dimer emission peak $(680 \mathrm{~nm})$ was present in all the cases, irrespective of the counterion (Figure 1b). However, the intensity of the dimer emission peak differs with counterion although any determinant role of counterion toward the dimer emission peak can be excluded. In addition to the counterion, the nature of the heteroaromatic moiety may also influence the dimer emission (see Figure S1).

The ratio of the intensities of the two emissions did not change when the excitation wavelength was varied from 450 to $580 \mathrm{~nm}$. Also, the excitation spectra acquired at 587 and 679 $\mathrm{nm}$ are similar (Figure 1c). The latter double peak corresponds to the absorption spectra of monomer and dimer. From this observation, it can be concluded that we are dealing with an excited state process.

The absorption spectrum and excitation spectra obtained for both monomer and dimer emissions resemble each other, but the ratio of the intensities of the two bands differs. While in absorption the main band is more intense than the dimer band, in excitation the bands have similar intensities. The disparity 

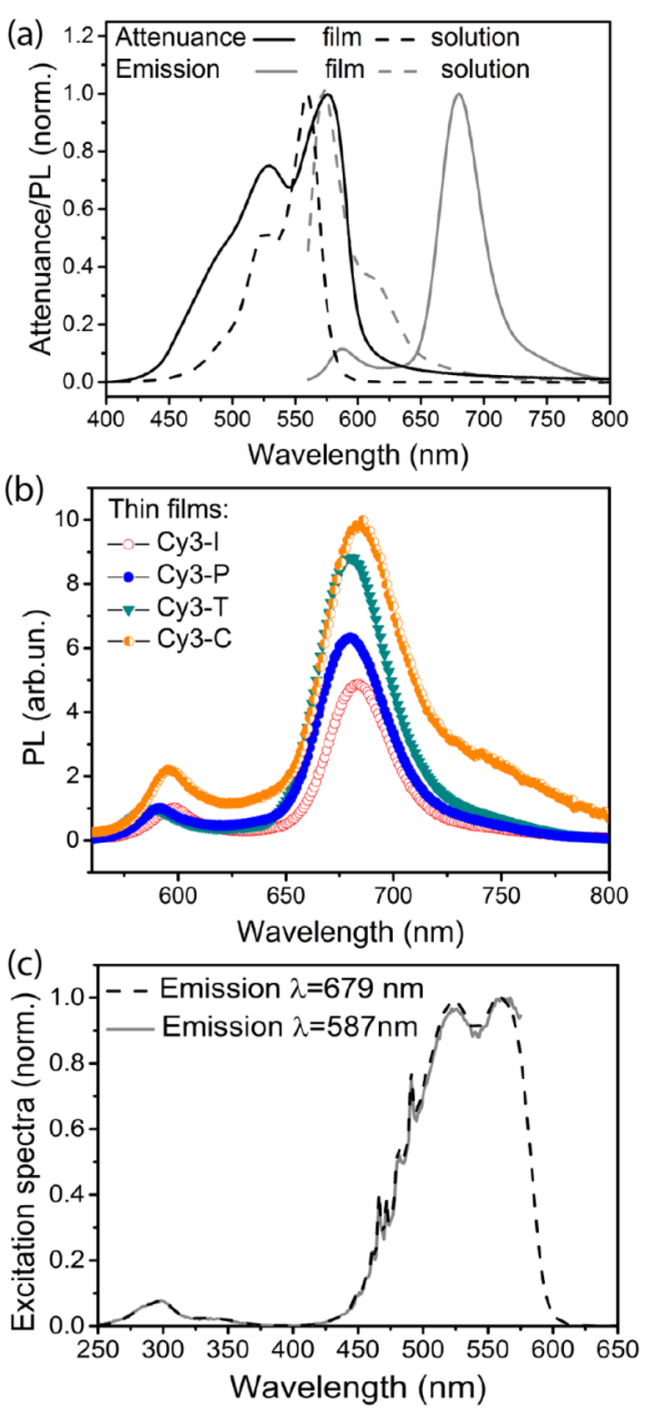

Figure 1. Attenuance and PL spectra for Cy3-T in CB solution and spin-coated film (a). PL spectra from cyanine films with different counterions (b). Excitation spectra for Cy3-T films when emission was monitored at the monomer $(587 \mathrm{~nm})$ and dimer $(679 \mathrm{~nm})$ peak (c).

between the absorption and excitation spectra arises because UV-vis measurements (Figure 1a) record a ground state absorption process while the excitation spectra (Figure 1c) are acquired in the excited state. The latter case also probes energy exchange between both monomer and dimer. This will show different amounts of monomer and dimer species, on comparing the excitation and absorption spectra. As the population of the emissive dimer species is not the only deactivation pathway, the fraction of emissive dimers cannot be quantified. But direct excitation of the dimer emission cannot be observed, indicating a low fraction of the latter. Although dimer emission (in parallel arrangement) is generally optically forbidden, it can be hypothesized that the distortion of the molecule can break the selection rule that forbids transition, giving rise to an emission. Emission from the trans-triplet state of a structurally very similar dye has been measured in methanol at $77 \mathrm{~K}$ and found to be around $722 \mathrm{~nm} .^{22}$ This emission is significantly more red-shifted than the dimer emission in this work and represents one of many arguments against triplet emission as an explanation.
For all dyes, at temperatures between 77 and $300 \mathrm{~K}$, no paramagnetic species were identified by ESR or LESR either in $g$ close to 2 (singlet) or 4 (triplet). However, the measurement of dye Cy3-T at $6.5 \mathrm{~K}$ under illumination showed the presence of two ESR signals attributed to cationic and anionic dyes. Again, no triplet signals could be observed ( $g$ close to 4 ). The signals were weak with $g=2.0026$ and line width of $0.6 \mathrm{mT}$ from the anion and $g=2.0023$ with line width of $2.3 \mathrm{mT}$ from the cation. The origin of the signals is attributed to charge transfer of the photoexcited electron or hole between dye molecules generating paramagnetic species, cations, and anions. This observation was possible due to the effects of low temperature. At $6.5 \mathrm{~K}$ the photogenerated charges have relatively long recombination times and thus are more stable. This result also indicates that no triplet state is generated.

Determining the effect of temperature on emission signals can assist in analyzing the spectra. For example, triplet population is generally a temperature-dependent phenomenon. ${ }^{23}$ In addition, cis-trans isomerization of the molecules is hindered at lower temperatures. Spectra were measured while the samples were cooled by adiabatic compression from room temperature $(\mathrm{RT})$ to $92 \mathrm{~K}$. Spectra were also taken during the warm-up phase back to RT and then during heating to $340 \mathrm{~K}$. The samples were excited at $480 \mathrm{~nm}$, and the two emission peaks at 600 and $680 \mathrm{~nm}$ were observed (Figure 2a). Upon cooling, in the range from 300 to $180 \mathrm{~K}$ both peaks increase in intensity. Between 180 and $92 \mathrm{~K}$, the monomer peak intensity increases while the second emission decreases (Figure 2a,b). The cooling-heating cycle shows some hysteresis. Upon heating, between 92 and $120 \mathrm{~K}$ (Figure 2c), both peaks initially decrease in intensity. The intermediate heating regime where the monomer emission decreases and the second peak emission increases are evident between 130 and $240 \mathrm{~K}$ (Figure 2d). Between 290 and $340 \mathrm{~K}$ again both peak intensities decrease in intensity (Figure 2e). Outside of the intermediate cooling and heating regime, the sample shows a regular temperature response: fluorescence increases with decreasing temperature because nonradiative processes become less effective. The unusual temperature response of the second emission in the intermediate temperature range is consistent with a model where thermal motion of the molecule annuls the geometrical restrictions that makes the $S 1^{\prime}-\mathrm{S} 0$ transition impossible. Alternatively, the energy transfer to the emissive species can also be temperature dependent. The regular monomer emission undergoes an irreversible red-shift during the cooling cycle. It was also observed that the monomer emission is composed of two peaks that we identified as $0-0$ and $0-1$ (vibrational) transitions. Upon cooling to $92 \mathrm{~K}$ (Figure 2c), the vibronic shoulder becomes stronger in intensity causing the red-shift. This effect requires further attention and could be related to an isomeric phase transition that the molecules undergo during thermal excursion favoring the 0-1 transition. The second emission remains unaffected.

Dimerization in thin films cannot be avoided by spin-coating the dye from more dilute solutions. Dimer formation could be reduced by dispersing the Cy3-P dye at different concentration of 2, 1, 0.5, 0.05, 0.02, and 0.01 wt \% in a PVME polymer matrix. Absorbance measured on the spin-coated films is shown in Figure S2. Reduced dimerization is observed in samples with lower dye content $(0.01$ and $0.02 \mathrm{wt} \%)$. The samples were placed into an integrating sphere and excited within the monomer absorption band. 

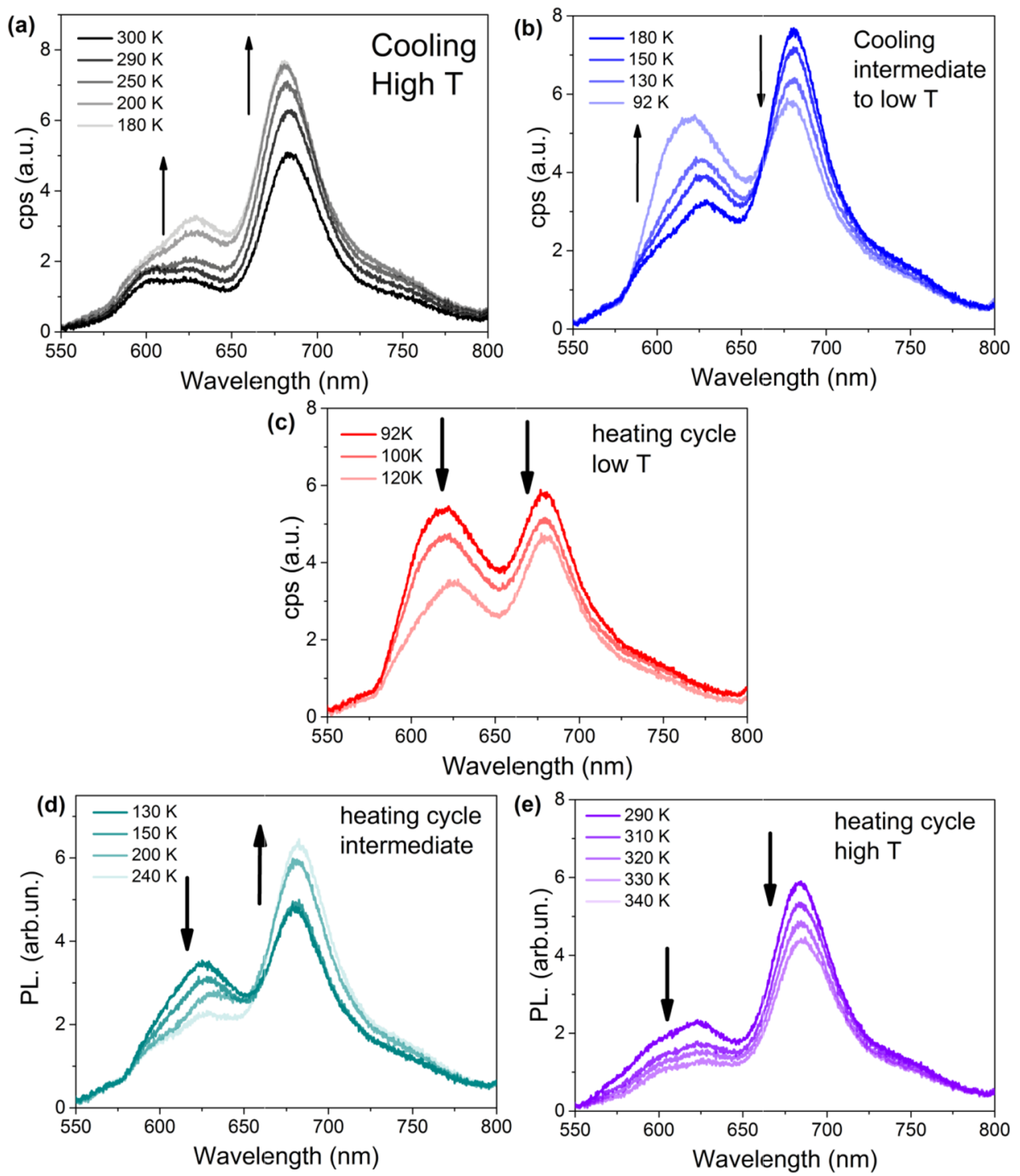

Figure 2. Temperature-dependent fluorescence studies of the cyanine films (Cy3-T) recorded from $92 \mathrm{~K}$ to room temperature on cooling ( $\mathrm{a}, \mathrm{b})$ and on heating $(c, d, e)$.

The intensity of the dimer emission $(680 \mathrm{~nm})$ increases with increasing dye concentration as shown in Figure 3a. At the lowest dye concentrations ( $0.01 \mathrm{wt} \%)$, significant monomer emission was detected, which decreases when increasing the dye concentration. Above $0.5 \mathrm{wt} \%$, monomer emission is fully quenched. The remaining small peak at $590 \mathrm{~nm}$ could originate from the vibronic peak or is due to energy exchange between dimer to monomer. At low dye concentrations, monomer emission is high because the monomers are not surrounded by dimers that would quench the emission. Increasing the dye concentration increases the probability for energy transfer from an excited monomer to a dimer, resulting in a strong dimer emission. When the emission intensity is normalized by the dye concentration, emission from both monomer and dimer is strongly concentration dependent in the low concentration regime, signifying the importance of dilution (Figure $3 \mathrm{~b}$ ). In the high concentration regime, the dimer emission is nearly constant. The fraction of dimers that are emissive remains constant with dye concentration. The deviation from proportionality with dye concentration above $1 \mathrm{wt} \%$ is due to $\mathrm{H}$-aggregate formation that quenches the emission further.
As hypothesized above, the reason for dimer emission could be the twisting of molecules against each other in the film. In that case, the excited states can obtain rotatory strength. In the $\mathrm{CD}$ spectrum, exciton coupling can be displayed as classic Cotton effect, with positive and negative contributions at different energies. ${ }^{24,25}$ The films described here show signatures from $\mathrm{H}$-aggregate and dimer configurations and are thus not easy to interpret (Figure 3c). At low dye concentrations, the signal is positive in the $\mathrm{H}$-aggregate and dimer absorption region. Increasing the dye concentration to $1 \mathrm{wt} \%$, the dimer signal turns negative, while the $\mathrm{H}$-aggregate has a bisignate signal with a positive contribution at lower wavelength and a negative contribution at higher wavelength (merging with the dimer signal). The samples with high dye concentration (2 wt $\%$ ) in the PVME matrix showed a strong negative signal (510 $\mathrm{nm}$ ) at the dimer peak position (Figure 3c) but no positive contribution from the $\mathrm{H}$-aggregate. This negative signal in the dimer position was observed irrespective of the matrix, i.e., when the dye was dispersed in a PMMA matrix (see Figure S3) during preparation of the thin films. Buss ${ }^{26}$ reported a similar observation when dispersing cyanines in $\gamma$-cyclodextrin in solution. With decreasing dimer concentration, the CD signal 


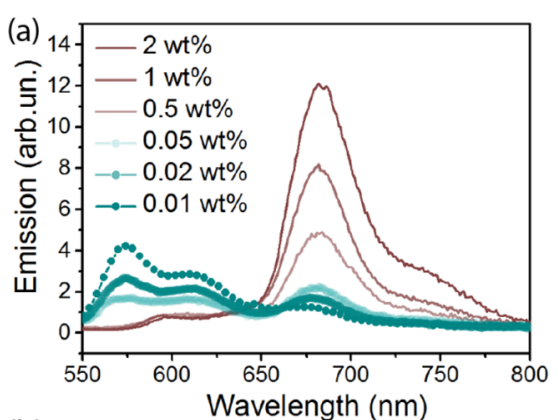

(b)
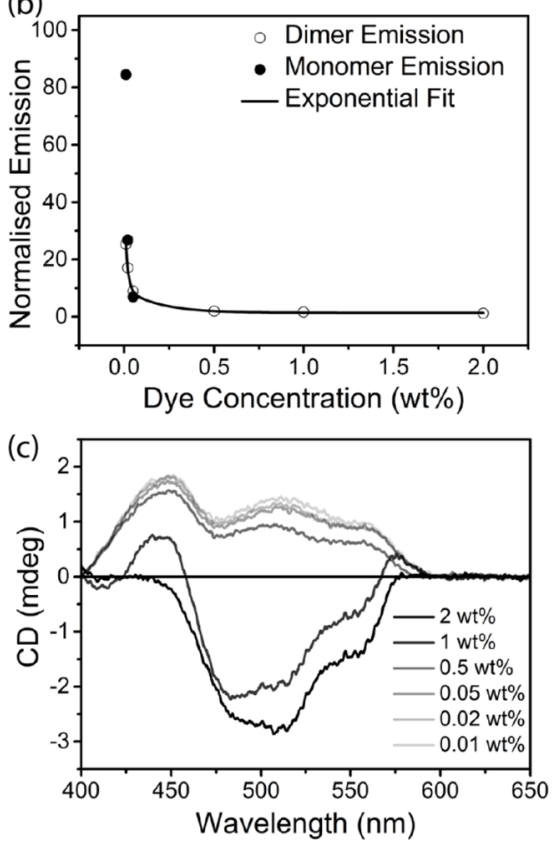

Figure 3. Emission spectra for different concentrations of Cy3-P dye dispersed in a PVME matrix (a). The dimer and monomer emission are normalized with the dye concentration in the PVME matrix (b). Circular dichroism (CD) spectra for different concentration of Cy3-P dye dispersed in the PVME matrix (c).

showed a transition from negative to positive apex. This behavior was explained by proposing that the dimers were trapped with a twisted sandwich configuration in the matrix. A similar observation here validates our hypothesis that dimers are twisted in oblique configuration in the films.

We can only speculate on the different molecular configurations of the dyes at different degrees of packing. However, the signals observed in the negative or positive apex of the dimer peak position indicate that some dimers are packed such that they become optically active, which results in different absorption of light in right-circular and left-circular directions. This difference in absorption also indicates that the net transition dipole moments are not parallel unlike the normal transition in the classical dimers. Therefore, a small resultant transition dipole moment results in absorption of light along with fluorescence.

An equally viable reason for the role of excimer is the twisting of molecules in the excited state. The difference between a twisted dimer and an excimer can be resolved with the following argument: If indeed a stable excimer (excited dimer) configuration existed, it would give rise to much broader and unstructured band. ${ }^{27}$ However, this is not observed in our study, as shown in Figure 3a where the dimer emission is narrow with a vibronic band in the film. This supports the role of molecular twisting which is preserved in films and not in solution.

The lifetime of the dimer emission peak was always longer than the monomer emission peak. For instance, in the case of Cy3-T films, the monomer shows a lifetime of $0.085 \mathrm{~ns}$, whereas the dimer shows a lifetime of $1.069 \mathrm{~ns}$. The lifetime of the dimer emission peak in Cy3-P was 0.668 ns (Figure 4).

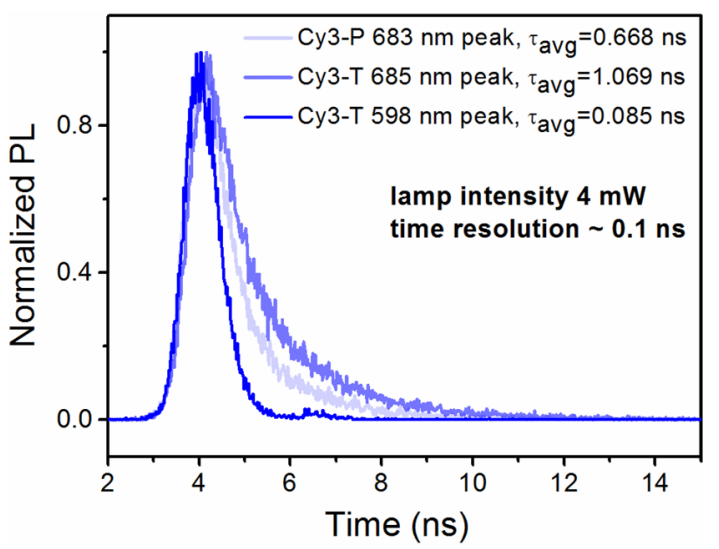

Figure 4. Lifetime measurement for the monomer and dimer emission from Cy3-T and Cy3-P spin-coated films.

Because of the limited resolution of the instrument (100 ps), the direct Cy3-P fluorescence could not be measured (lifetime around $50 \mathrm{ps}) .^{28}$ Thus, the lifetime of the dimer emission is approximately 1 order of magnitude longer than the monomer emission. The dimers are more stable in the presence of oxygen as detailed in the Supporting Information (see Figure S4).

Fluorescence quenching is a powerful tool to measure exciton diffusion lengths and also to assess whether the excitons are diffusive or localized species. ${ }^{29}$ Two experiments were performed on a model system for a doped dye film and a bilayer device. In the first experiment, the dye layer was blended with $\mathrm{NOPF}_{6}$ at different concentrations. ${ }^{30}$ In the second experiment, a $\mathrm{C}_{60}$ fluorescence quenching layer was deposited on top of dye layers of different thickness. The sample was illuminated from the dye side. Both materials have been shown to quench both emissions, but the dimer emission is expected to be quenched more efficiently, as during its longer lifetime it has a higher probability to react with the quenching site.

The results of the first experiments show that when the dye film is $\mathrm{p}$-doped at the lowest doping $\left(\mathrm{NOPF}_{6} / \mathrm{Cy} 3-\mathrm{P}=0.05\right)$, the dimer emission is already fully quenched, whereas the monomer emission is only fully quenched at $\mathrm{NOPF}_{6} / \mathrm{Cy} 3-\mathrm{P}=$ 1 (Figure 5a). In addition, the film morphology changes upon heavy doping. In the second experiment, the fraction of the unquenched emission of monomer to dimer increased as the dye film became thinner (Figure $5 b$ ).

This observation supports the proposal that the dimer emission is quenched more efficiently than the monomer emission. The second experiment suggests that the excited emitting dimer state is localized near the $\mathrm{C}_{60}$ interface while the monomer exciton is allowed to diffuse. As the dye film thickness increases (30 and $55 \mathrm{~nm}$ ), the fraction of quenched emitting dimers decreases. For very thick films $(100 \mathrm{~nm}$, not shown here), the light absorption region moved away from the dye $/ \mathrm{C}_{60}$ interface, and the emission behavior of pure Cy3 films was recovered. On the other hand, thinner films $(20 \mathrm{~nm})$ show a stronger decrease in dimer emission than monomer. The ratio 

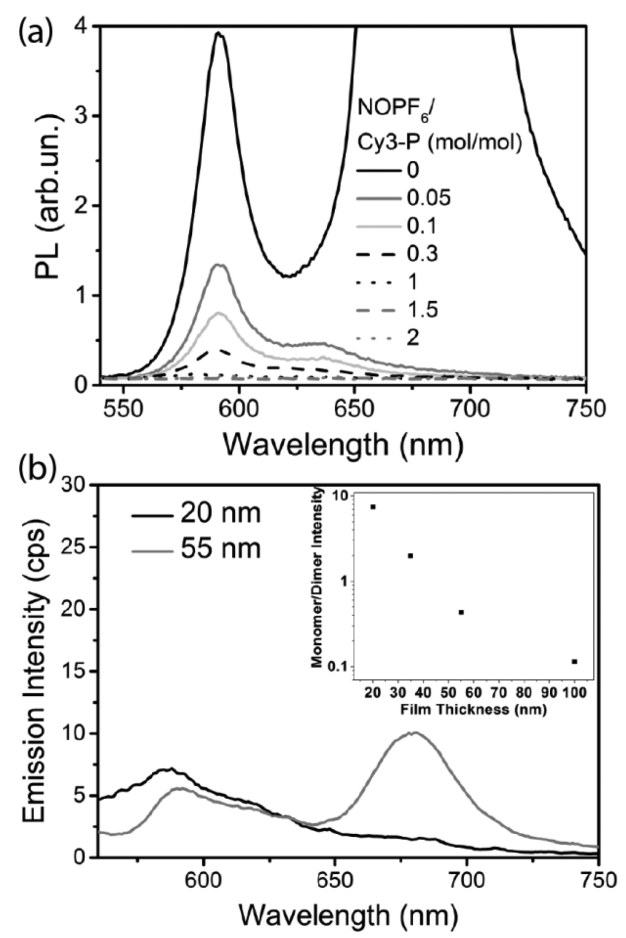

Figure 5. Blends of $\mathrm{NOPF}_{6} / \mathrm{Cy} 3-\mathrm{P}$ show that the dimer is quenched more efficiently than the monomer emission (a). Nonquenchable emission intensity from Cy3-P films spin-coated on $\mathrm{C}_{60}$ decreases with increase in the film thickness. The ratio of monomer to dimer intensity with film thickness is shown in the inset (b).

of monomer to dimer unquenched emission (as shown in the inset, Figure 5b) first decreases exponentially as a function of dye film thickness but then approaches a maximum value. The data are consistent with a model where the second emission is quenched very efficiently only from localized sites next to the $\mathrm{C}_{60}$ interface, while the monomer emission is more efficiently quenched over a large distance through the film (exciton diffusion). At $100 \mathrm{~nm}$ (not shown here) dye film thickness, the sample shows the pure dye layer behavior as no light is absorbed near the quenching sites.

The typical quantum efficiency in a thin cyanine film with only monomer emission is around $1.5 \%$, while the dimer emission alone contributes $8.5 \% .^{31}$ Also, the dimer emission decreases exponentially with the dye concentration in solid matrices (Figure 3b). Given the assumption that there is a low fraction of emitting dimer species, concentration quenching can be overcome leading to higher quantum yield. We propose that this sets a lower limit to the concentration of emitting dimer species. Assuming half of the dye molecules are assembled into a dimer, and the transfer rate from monomer to dimers as well as fluorescence quantum efficiency is near $100 \%$, a high estimate of the fraction of emissive dimers could be $10 \%$.

\section{CONCLUSIONS}

A red-shifted PL band in thin film samples of a cyanine dye was observed and arises from a low fraction of dimers that are present in a twisted configuration. Without this twisted configuration the net transition dipole moment would be zero. The existence of these states is confirmed by $\mathrm{CD}$ measurements. Dispersing either monomer or dimer in a matrix at low concentration can be a means to overcome selfquenching resulting in strong fluorescence, as observed in this study. Temperature-dependent emission shows that dimer-tomonomer emission is dominant at room temperature and weak at low temperatures. Also, the lifetime of the dimer in the excited state is longer than for the monomer, more stable in the presence of oxygen and quenchable at room temperature, which satisfies the most important prerequisites for application in devices. We propose that engineering the molecular stacking in the film and harvesting this strong fluorescence as demonstrated in this study can overcome the limitation of ACQ to improve the performance of the devices built with cyanine dyes.

\section{ASSOCIATED CONTENT}

S Supporting Information

The Supporting Information is available free of charge on the ACS Publications website at DOI: 10.1021/acs.jpcc.7b01412.

Nonemissive dimers, attenuance with dye concentration, stability of monomer and dimer emission (PDF)

\section{AUTHOR INFORMATION}

\section{Corresponding Author}

*E-mail: jakob.heier@empa.ch (J.H.).

\section{ORCID}

Sergii Yakunin: 0000-0002-6409-0565

Marcus Vinícius Gonçalves Vismara: 0000-0001-8616-9701

Roland Hany: 0000-0003-0569-119X

Maksym V. Kovalenko: 0000-0002-6396-8938

Jakob Heier: 0000-0003-2189-3162

\section{Notes}

The authors declare no competing financial interest.

\section{ACKNOWLEDGMENTS}

We gratefully thank Prof. Dr. Raffaele Mezzenga and Ms. Wenjie Sun from ETH, Zürich, for extending their support to access the CD spectroscopy in their lab. We acknowledge the financial support received from the Swiss National Science Foundation (SNF) Grants 200021-157135 and 200021-144120 for conducting this work. C.F.O.G. acknowledges the financial support from FAPESP (2013/07296-2) and CNPq.

\section{REFERENCES}

(1) Kietzke, T. Recent Advances in Organic Solar Cells. Adv. OptoElectron. 2007, 2007, 1-15.

(2) Tani, T. Photographic Science Advances in Nanoparticles, JAggregates, Dye Sensitization, and Organic Devices; Oxford University Press: Oxford, UK, 2011.

(3) Varghese, S.; Das, S. Role of Molecular Packing in Determining Solid-State Optical Properties of П-Conjugated Materials. J. Phys. Chem. Lett. 2011, 2, 863-873.

(4) Kasha, M.; Rawls, H. R.; Ashraf El-Bayoumi, M. The Exciton Model in Molecular Spectroscopy. Pure Appl. Chem. 1965, 11, 371392.

(5) Chambers, R. W.; Kajiwara, T.; Kearns, D. R. Effect of Dimer Formation on the Electronic Absorption and Emission Spectra of Ionic Dyes. Rhodamines and Other Common Dyes. J. Phys. Chem. 1974, 78, 380-387.

(6) Rösch, U.; Yao, S.; Wortmann, R.; Würthner, F. Fluorescent HAggregates of Merocyanine Dyes. Angew. Chem., Int. Ed. 2006, 45, 7026-7030.

(7) Würthner, F.; Yao, S. Dipolar Dye Aggregates: A Problem for Nonlinear Optics, but a Chance for Supramolecular Chemistry. Angew. Chem., Int. Ed. 2000, 39, 1978-1981. 
(8) Zeena, S.; Thomas, K. G. Conformational Switching and Exciton Interactions in Hemicyanine-Based Bichromophores. J. Am. Chem. Soc. 2001, 123, 7859-7865.

(9) Matsui, M.; Ando, S.; Fukushima, M.; Shibata, T.; Kubota, Y.; Funabiki, K. Fluorescence Properties of Indolenium Carbocyanine Dyes in Solid State. Tetrahedron 2015, 71, 3528-3534.

(10) Chen, Y.; Lee, B.; Fu, D.; Podzorov, V. The Origin of a $650 \mathrm{Nm}$ Photoluminescence Band in Rubrene. Adv. Mater. (Weinheim, Ger.) 2011, 23, 5370-5375.

(11) Würthner, F.; Sens, R.; Etzbach, K.-H.; Seybold, G. Design, Synthesis, and Evaluation of a Dye Library: Glass-Forming and SolidState Luminescent Merocyanines for Functional Materials. Angew. Chem., Int. Ed. 1999, 38, 1649-1652.

(12) Ferreira, L. F. V.; Oliveira, A. S.; Wilkinson, F.; Worrall, D. Photophysics of Cyanine Dyes on Surfaces. A New Emission from Aggregates of 2,2'-Cyanines Adsorbed onto Microcrystalline Cellulose. J. Chem. Soc., Faraday Trans. 1996, 92, 1217-1225.

(13) Ferrer, M. L.; del Monte, F.; Levy, D. Rhodamine 19 Fluorescent Dimers Resulting from Dye Aggregation on the Porous Surface of Sol-Gel Silica Glasses. Langmuir 2003, 19, 2782-2786.

(14) Chen, Z. J.; Lohr, A.; Saha-Möller, C. R.; Wurthner, F. SelfAssembled Pi-Stacks of Functional Dyes in Solution: Structural and Thermodynamic Features. Chem. Soc. Rev. 2009, 38, 564-584.

(15) Chibisov, A. K. Photonics of Dimers of Cyanine Dyes. High Energy Chem. 2007, 41, 200-209.

(16) Ferencz, A.; Neher, D.; Schulze, M.; Wegner, G.; Viaene, L.; De Schryver, F. C. Synthesis and Spectroscopic Properties of Phthalocyanine Dimers in Solution. Chem. Phys. Lett. 1995, 245, $23-29$.

(17) Asano-Someda, M.; Ichino, T.; Kaizu, Y. Triplet-Triplet Intramolecular Energy Transfer in a Covalently Linked Copper(Ii) Porphyrin-Free Base Porphyrin Hybrid Dimer: A Time-Resolved Esr Study. J. Phys. Chem. A 1997, 101, 4484-4490.

(18) Sorokin, A. V.; Gnap, B. A.; Bespalova, I. I.; Yefimova, S. L.; Malyukin, Y. V. Configuration of Organic Dye Excimers in Nanoporous $\mathrm{SiO}_{2}$ Matrices. J. Lumin. 2016, 179, 171-177.

(19) Mei, J.; Hong, Y.; Lam, J. W. Y.; Qin, A.; Tang, Y.; Tang, B. Z. Aggregation-Induced Emission: The Whole Is More Brilliant Than the Parts. Adv. Mater. (Weinheim, Ger.) 2014, 26, 5429-5479.

(20) Jenatsch, S.; Wang, L.; Bulloni, M.; Véron, A. C.; Ruhstaller, B.; Altazin, S.; Nüesch, F.; Hany, R. Doping Evolution and Junction Formation in Stacked Cyanine Dye Light-Emitting Electrochemical Cells. ACS Appl. Mater. Interfaces 2016, 8, 6554-6562.

(21) de Mello, J. C.; Wittmann, H. F.; Friend, R. H. An Improved Experimental Determination of External Photoluminescence Quantum Efficiency. Adv. Mater. (Weinheim, Ger.) 1997, 9, 230-232.

(22) Jia, K.; Wan, Y.; Xia, A.; Li, S.; Gong, F.; Yang, G. Characterization of Photoinduced Isomerization and Intersystem Crossing of the Cyanine Dye Cy3. J. Phys. Chem. A 2007, 111, $1593-1597$.

(23) Kohler, A.; Bassler, H. Triplet States in Organic Semiconductors. Mater. Sci. Eng., R 2009, 66, 71-109.

(24) Mason, S. F. Non-Anomalous Absolute Configuration of 1,5Disubstituted 9,10-Dihydro-9,10-Bridged Anthracenes. J. Chem. Soc., Chem. Commun. 1973, 239-241.

(25) Berova, N.; Bari, L. D.; Pescitelli, G. Application of Electronic Circular Dichroism in Configurational and Conformational Analysis of Organic Compounds. Chem. Soc. Rev. 2007, 36, 914-931.

(26) Buss, V. Circular Dichroism of a Chiral Cyanine Dye Dimer Trapped in Г-Cyclodextrin. Angew. Chem., Int. Ed. Engl. 1991, 30, 869-870.

(27) Sims, M.; Bradley, D. D. C.; Ariu, M.; Koeberg, M.; Asimakis, A.; Grell, M.; Lidzey, D. G. Understanding the Origin of the $535 \mathrm{Nm}$ Emission Band in Oxidized Poly(9,9-Dioctylfluorene): The Essential Role of Inter-Chain/Inter-Segment Interactions. Adv. Funct. Mater. 2004, 14, 765-781.

(28) De Jonghe-Risse, J.; Heier, J.; Nuesch, F.; Moser, J.-E. Ultrafast Charge Transfer in Solid-State Films of Pristine Cyanine Borate and Blends with Fullerene. J. Mater. Chem. A 2015, 3, 10935-10941.
(29) Lunt, R. R.; Giebink, N. C.; Belak, A. A.; Benziger, J. B.; Forrest, S. R. Exciton Diffusion Lengths of Organic Semiconductor Thin Films Measured by Spectrally Resolved Photoluminescence Quenching. J. Appl. Phys. (Melville, NY, U. S.) 2009, 105, 053711-7.

(30) Fan, B.; de Castro, F. A.; Heier, J.; Hany, R.; Nüesch, F. High Performing Doped Cyanine Bilayer Solar Cell. Org. Electron. 2010, 11, $583-588$.

(31) Jenatsch, S.; Wang, L.; Leclaire, N.; Hack, E.; Steim, R.; Anantharaman, S. B; Heier, J.; Ruhstaller, B.; Penninck, L.; Nüesch, F.; Hany, R. Submitted for publication. 